\title{
Laro: Isang estratehiya sa pagtuturo ng numeracy
}

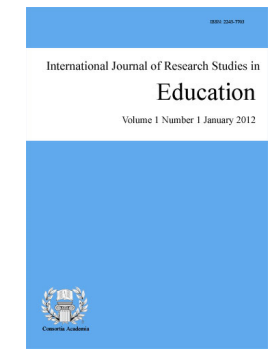

ISSN: 2243-7703 Online ISSN: 2243-7711

Accepted: 29 May 2019

\section{Abstract}

This study shows the effectiveness of Game as a strategy in teaching Numeracy in Kindergarten. There are forty- seven (47) respondents from the Kindergarten of Biton Elementary School, Magallanes, Sorsogon. Descriptive developmental is used in the study. Pre-test and post-test tests are instruments used to determine the level of skills of the students in Numeracy. Frequency, mean, weighted mean and chi-square are used as a statistical tool. Based on the outcome of the study findings are as follows. The levels of students in Hand Game Addition, Hand Game Subtraction both have the Description of Beginning. Also the Lift the Bowl Addition and Lift the Bowl Subtraction have the same description which is beginning. The games used were effective in developing numeracy skills. There is an important difference in numeracy skills of students in pre-test and posttest using the game, the null hypothesis was rejected. The proposed book for Kindergarten instruction is to be done or obeyed by the teacher. The conclusion was made that the skill level of students in Hand game Addition, Hand game Subtraction, Lift the Bowl Addition and Lift the Bowl subtraction are all at the Beginning level. The result of Numeracy's teaching has been effective with the Hand Game Addition, Hand Game Subtraction, Lift the Bowl Addition and Lift the Bowl Subtraction to Kindergarten students. The numeracy skills of the pupils increase because of the use of Hand Game and Lift the Bowl as strategy. A book was developed for teaching Numeracy in Kindergarten. From these conclusions, it is recommended that Kindergarten teachers should try to enhance the Numeracy skills of the pupils by stimulating their interest in the use of various approaches and teaching strategies.

Keywords: estratehiya; numeracy; kindergarten; pamamaraan; lift the bowl; hand game 


\section{Laro: Isang estratehiya sa pagtuturo ng numeracy}

\section{Introduction}

Ang edukasyon ay isang anyo ng pag-aaral kung saan ang kaalaman, kasanayan, at mga gawi ng isang pangkat ng mga tao ay inililipat mula sa isang henerasyon at sa susunod pa sa pamamagitan ng pagtuturo, pagsasanay, at riserts. Ang edukasyon ay madalas maganap sa ilalim ng paggabay ng iba, gaya ng ating mga magulang, kaibigan, kalaro, kapamilya at siyempre ng ating butihing guro. Dito sa Pilipinas ang edukasyon ay nahahati sa tatlo, ang elementarya, sekundarya at tersarya. Ang Kindergarten ay kasama sa antas elementarya na kung saan ang mga batang may edad lima ay hinihikayat na pumasok sa paaralan upang sila ay maihanda sa kanilang pagpasok sa unang baitang o Grade I (Belvez, 2005).

Ayon sa "DepEd Order No. 37 s. 2011" ang Kindergarten ay isang mandatory at compulsory na siyang maghahanda sa mga batang limang taong gulang sa isang pormal na edukasyon sa elementarya. Ang Batas na ito ay ibinatay sa Goal ng Kagawaran ng Edukasyon na "Education for All (EFA)". Ayon kay DepEd Secretary Br. Armin A. Luistro ang batas na ito ay nagbibigay ng pagkakataon sa mga bata na magkaroon ng pantay- pantay na pagkakataon upang makapag-aral sa kindergarten na siyang huhubog sa kanilang kabuuan (Code of Ethics for Teachers).

Ang programa ng Kindergarten ay tumutulong upang ang mga batang nasa edad na limang taong gulang ay makapag-galugad, matututong makipagkapwa-tao at matutunan ang mga aralin. Nililinang dito ang pagmamahal ng mga bata sa pagkatuto. Kailangan ng mga guro na humanap ng paraan at mga estratehiya upang makuha ang interes ng mga mag-aaral upang sila ay matuto lalong lalo na sa asignaturang Numeracy (Tindugan, 2014).

Ang pagtuturo ay isang larangan ng konsiderableng antas ng kahusayan, pagkamalikhain, pagiging epektibo at mapamaraan ng gurong nangangasiwa upang maikintal nang maayos at maunlad ang mga kaalamang kailangan ng mga mag-aaral na siyang bubuo sa isang mapayapa at masaganang lipunan. Pagtuturong siyang huhubog sa kabuuang pag-unlad intelektwal.

Ang pagtuturo ay isang napakahirap na gawain. Hindi sapat na ikaw ay magtuturo lamang, kailangan na ito'y naiintindihan ng iyong mga mag-aaral upang maging makabuluhan ang iyong pagtuturo. Ang mga guro ay patuloy na nag-aaral kung ano ang mga angkop na estratehiya sa pagtuturo ng mga aralin upang ang mga mag-aaral ay mahikayat na mag-aral at matutunan ang kanilang mga aralin. Ang isang guro ay nagsisikap na magkaroon ng baryedad ng mga gawain sa klase upang maiwasan ang pagkainip o pagkabagot, pagkasawa at panlalamig ng sigasig ng mga mag-aaral (Belvez, 2005).

Isa sa mga layunin ng mga guro ay maibahagi ang kanilang kaalaman ng buong-buo sa mga mag-aaral ang mga bagay na dapat nilang malaman. Ito'y isang napakahirap na gawain lalo na kung ang iyong mag-aaral ay walang interes sa inyong mga aralin. Kaya ang mga guro ay pilit humahanap ng mga estratehiya, metodo at kung ano ano pa upang ang kanilang mga aralin ay maging kawili-wili sa kanilang mga mag-aaral. Sa panahon ngayon ang mga kabataan ay nawiwili sa panonood ng telebisyon, computer at paglalaro na nagreresulta sa pagpapabaya sa kanilang mga aralin. Isang napakalaking suliranin sa mga guro na makuha ang atensyon at interes ng kanilang mag-aaral. Kinakailangang mag-isip ng mga guro ng iba't ibang estratehiya na upang makuha ang pansin ng mga mag-aaral. Upang higit na mahikayat ang mga mag-aral kinakailangan ng isang guro na matutunan ang iba't ibang mga estratehiya sa pagtuturo. Sinasabing ang asignaturang Numeracy o Matematika ay isang mahirap at kabagotbagot na asignatura para sa mga mag-aaral kung kaya kinakailangang alamin ang iba't ibang estratehiya sa pagtuturo ng asignaturang ito na siyang magiging daan sa isang kasiya-siyang talakayan at pagkatuto ng mga bata. Sa pagtuturo kinakailangang gawing kawili-wili, ang mga aralin sa kasanayang Numeracy upang mahikayat ang mga mag-aaral na pag-aralan ito. Ang mga guro ay kinakailangan na mayroong sapat na kaalaman sa kanyang mga itinuturo. Kinakailangang gumamit ng mga angkop na mga estratehiya upang ang 
pagtuturo ay magkaroon ng magandang resulta (Nolasco, 2012).

Ang Kindergarten ngayon ay sakop ng K to 12 kurikulum na kung saan ang pagtuturo ay idinadaan sa isang laro. Ayon sa mga pag-aaral na isinagawa, ang mga batang limang taong gulang ay natututong mag-isa at mas mabilis niyang natutunan kung paano makikagkapwa-tao. Nagkakaroon na sila ng mga kaibigan at nagkakaroon sila ng tiwala sa kanilang sarili. Sila ay mahilig maglaro at gustong-gusto nilang matuto. Habang ang mga bata ay naglalaro lumalawak ang kanilang mga kaalaman dahil sila ay natututo habang sila ay naglalaro (Orfiano, 2013).

Sa naging obserbasyon ng mananaliksik sa kanilang paaralang tinuturuan hindi maikakaila na ang addition at subtraction ay sadyang mahirap na aralin lalong lalo na sa kindergarten na mag-aaral. Ang ganitong kahirapan at problema ay kinakailangan bigyang pansin upang ang mga mag-aaral ay hindi mahuli sa kanilang mga aralin sa susunod na baitang. Kinakailangan gumamit ang guro ng iba't ibang estratehiya at pamamaraan upang mas maintindihan ng mga bata kung paano gawin ang kasanayang addition at subtraction. Ito ang nag-udyok sa mananaliksik na isagawa ang nabanggit na pag-aaral (Sandigan, 2010).

\subsection{Paglalahad Ng Suliranin}

Ang pag-aaral na ito ay naglalayong masagutan ang mga sumusunod:

1. Ano ang antas ng kasanayan ng mga batang mag-aaral sa kindergarten gamit ang laro na pamamaraan batay sa pre-test?

2. Gaano kaepektibo ang laro sa paglinang ng kasanayan sa Numeracy ng mga batang kindergarten batay sa posttest?

3. May mahalaga bang pagkakaiba sa antas ng kasanayan sa Numeracy ng mga mag-aaral batay sa pre-test at posttest.

4. Ano ang mga mungkahing gawain sa pagpapaunlad ng kasanayan ng mga Kinder sa Numeracy.

\section{Pamamaraang ginamit}

Ang pag-aaral na ito ay gumamit ng desinyong deskriktibong-debelopmental na pamamaraan upang masuri ang datos o impormasyon. Ang respondent ng pag-aaral na ito ay binubuo ng 47 na mag-aaral sa Kindegarten ng Biton Elementary School. Dalawampu ay lalaki at dalawampu't pito ay babae. Ang pag-aaral na ito ay natamo sa pamamagitan ng pagsusulit na gawa mismo ng mananaliksik. Inihanda ang mga bata sa isang laro na Lift the Bowl at Hand Game. Ang mga pagsusulit ay gawa mismo ng mananaliksik (teacher-made). Isinagawa ang pre-test at posttest.

\section{Mga natuklasan}

Inilalahad dito ang interpretasyon ng mga datos na nakalap mula sa kalahok na mag-aaral o respondents. Gumamit ng talahanayan upang maipakita ang mga datos at interpretasyon, ito ay makikita ayon sa mga sumusunod: (1) Ano ang antas ng kasanayan ng mga batang mag-aaral sa kindergarten gamit ang laro na pamamaraan batay sa pre-test (2) Gaano kaepektibo ang laro sa paglinang ng kasanayan sa numeracy ng mga batang kindergarten ayon sa posttest, at (3) May mahalaga bang pagkakaiba sa antas sa numeracy ng mga magaaral ayon sa pre-test at posttest?

\subsection{Antas ng kasanayan ng mga batang mag-aaral sa kindergarten gamit ang laro na pamamaraan batay sa pre-test}

Ang antas ng kasanayan ng mga batang mag-aaral sa Kindergarten gamit ang laro na pamamaraan batay sa 
Huevos, M., \& Marbella, F.

pre-test ay Beginning.

Makikita sa Talahanayan 1 ang antas ng kasanayan ng mga mag-aaral sa Hand game addition at subtraction, Lift the bowl addition at subtraction ayon sa pre-test. Sa Hand game addition, 74.11 ang mean ng kasanayan na may deskripsyon na beginning. 70.89 naman sa Hand game Subtraction na may desckripsyon na beginning samantalang 70.18 naman ang mean ng kasanayan sa Lift the Bowl Addition na may deskripsyon na beginning. Sa Lift the Bowl subtraction, 71.43 ang mean ng kasanayan na may deskripson na beginning.

\section{Talahanayan 1}

Antas ng kasanayan ng mga mag-aaral sa kindergarten gamit ang laro na pamamaraan batay sa pre-test

\begin{tabular}{|c|c|c|}
\hline Laro & Mean ng kasanayan & $\begin{array}{c}\text { Diskripsiyon } \\
\text { Antas ng Kaalaman }\end{array}$ \\
\hline $\begin{array}{l}\text { A. Hand Game } \\
\text { (Addition) }\end{array}$ & 74.11 & Beginning \\
\hline $\begin{array}{l}\text { B. Hand Game } \\
\text { (Subtraction) }\end{array}$ & 70.89 & Beginning \\
\hline $\begin{array}{l}\text { C. Lift the Bowl } \\
\text { (Addition) }\end{array}$ & 70.18 & Beginning \\
\hline \multirow{2}{*}{$\begin{array}{l}\text { D. Lift the Bowl } \\
\text { (Subtraction) }\end{array}$} & 71.43 & Beginning \\
\hline & 71.65 & Beginning \\
\hline
\end{tabular}

Tala. Bilang karagdagan, makikita din sa talahanayan na ang kabuuang mean ng kasanayan ng mga mag-aaral sa kindergarten ay 71.65 na nasa beginning na antas.

Ang antas ng kasanayan ng mga batang mag-aaral sa kindergarten gamit ang laro bilang pamamaraan batay sa pre-test ay beginning. Dahil sa resultang ito masasabing nangangailangan ng estratehiya upang tumaas ang antas ng kasanayan sa Numeracy. Malinaw na implikasyon ng resultang lumabas sa pag-aaral na ito, na kinakailangang gumamit ng estratehiya sa pagtuturo ng Addition at Subtraction upang tumaas ang antas ng kasanayan ng mga mag-aaral sa Kindergarten.

Ang guro, dahil sila ang direktang magtuturo ng iba't ibang konsepto sa kung ano ba talaga ang Numeracy. Kailangan na magbigay ng iba't-ibang estratehiya upang ang pagtuturo ng Numeracy ay maging kawili-wili at hindi nakababagot sa mga mag-aaral. Kailangan na ang kapaligiran ay isang maayos at kaaya-ayang tingnan. Ang kapaligiran ay dapat na napapaligiran ng mga bagay na may kinalaman sa Numeracy na siyang titingnan ng mga bata upang ito'y kanyang mapag-aralan. Kailangan na ang mga batang ito ay maakit na pag-aralan ang Numeracy.

Mayroong relasyon ang performans ng mga mag-aaral at estratehiya sa pagtuturo na ginagamit ng mga guro. Ang lebel ng pagkatuto ng mga bata ay hindi nakadepende sa isang estratehiya lamang kung hindi sa iba't ibang estratehiya na naaangkop sa mga mag-aaral (Vargas, 1989).

\subsection{Kabisaan ng laro sa paglinang ng kasanayan sa numeracy ng mga batang kindergarten ayon sa posttest}

Makikita sa Talahanayan 2 ang antas ng kasanayan ng mga mag-aaral sa Hand game Addition at Subtraction, Lift the bowl Addition at Subtraction ayon sa posttest. Makikita din ang bahagdan ng itinaas ng antas ng kasanayan nila pakatapos ng post-test. Sa Hand Game Addition, 93.57 ang mean ng kasanayan na may deskripsyon na Advanced. Mataas ito ng 26\% sa resulta ng pre-test. 84.64 naman sa Hand game Subtraction na may deskripsyon na Proficient samantalang 93.39 naman ang mean ng kasanayan sa Lift the Bowl Addition na may deskripsyon na Advanced. Tumaas ng 19\% at 33\% ang resulta nito kaysa pre-test. Sa Lift the Bowl subtraction, 83.75 ang mean ng kasanayan na may deskripsyon na Approaching Proficiency. Mataas ito ng $17 \%$ kaysa pre-test.

Bilang karagdagan, makikita din sa talahanayan na ang kabuuang mean ng kasanayan ng mga mag-aaral sa kindergarten ay 88.84 na nasa Proficient na antas. Ito ay $24 \%$ na mataas kumpara sa kabuuang resulta sa pre-test 
ng mga mag-aaral. Nasa advanced ang antas ng kasanayan ng Lift the Bowl addition dahil ito ay tumaas ng 23.21 samantalang nasa Approaching proficiency ang Lift the Subtraction dahil ito ay nasa 12.32 lamang. Ang implikasyon ng resultang lumabas sa posttest ay epektibo ang laro sa paglinang ng kasanayan sa Numeracy ng mga bata. Tumaas ang resulta dahil ang mga gawain ay ginamitan ng estratehiya. Sa paggamit ng estratehiya nagkakaroon ng pagtaas sa antas ng kasanayan.

\section{Talahanayan 2}

Kabisaan ng laro sa paglinang ng kasanayan sa numeracy ng mga batang kindergarten ayon sa posttest

\begin{tabular}{|c|c|c|c|c|c|}
\hline Laro & $\begin{array}{l}\text { Mean } n \\
\text { Pre-te }\end{array}$ & $\begin{array}{l}\text { sanayan } \\
\text { pst test }\end{array}$ & $\begin{array}{c}\text { Diskripsiyon } \\
\text { Antas ng Kaalaman }\end{array}$ & $\begin{array}{l}\text { Difference from } \\
\text { the Pre test }\end{array}$ & $\begin{array}{l}\text { Rate of } \\
\text { Increase }\end{array}$ \\
\hline $\begin{array}{l}\text { A. Hand Game } \\
\text { (Addition) }\end{array}$ & 74.11 & 93.57 & Advanced & 19.46 & $26 \%$ \\
\hline $\begin{array}{l}\text { B. Hand Game } \\
\text { (Subtraction) }\end{array}$ & 70.89 & 84.64 & Proficient & 13.75 & $19 \%$ \\
\hline $\begin{array}{l}\text { C. Lift the Bowl } \\
\text { (Addition) }\end{array}$ & 70.18 & 93.39 & Advanced & 23.21 & $33 \%$ \\
\hline $\begin{array}{l}\text { D. Lift the Bowl } \\
\text { (Subtraction) }\end{array}$ & 71.43 & 83.75 & Approaching Proficiency & 12.32 & $17 \%$ \\
\hline Kabuuan & 71.65 & 88.44 & Proficient & 17.19 & $24 \%$ \\
\hline
\end{tabular}

Sa pagtuturo ng Matematika gumagamit siya ng "multimedia approach". Ginamit niya ang computer-aided instruction" sa kanyang mag-aaral sa Matematika IV at ito ay nagbigay ng magandang resulta. Nagpapatunay lamang ito na kinakailangan na gumamit ng estratehiya sa pagtuturo (Sandigan, 2010). Ang paggamit ng "game-based learning" o GBL ay nagreresulta sa pagkakaroon ng lakas ng loob ng mga mag-aaral. Ito ay nagbibigay ng ng mas mataas na antas ng kasanayan sa paggamit ng "paper-based setting”. Ang mga gumagamit ng GBL o laro ay mas nakakakuha ng mas mataas na antas ng kasanayan (Ku, Chen, \& Wu, 2014).

\subsection{Pagkakaiba sa antas sa numeracy ng mga mag-aaral ayon sa pre-test at posttest}

Ang pagkakaiba sa antas sa Numeracy ng mga mag- aaral batay sa pre-test at posttest sa Hand Game Addition, Hand Game Subtraction, Lift the Bowl Addition at Lift the Bowl Subtraction at ng kanilang kabuuang kasanayan sa numeracy ay makikita sa Talahanayan 3.

\section{Talahanayan 3}

Pagkakaiba sa antas sa numeracy ng mga mag-aaral ayon sa pre-test at posttest

\begin{tabular}{lccccc}
\hline \multirow{2}{*}{ Statistical Bases } & \multicolumn{4}{c}{ Statistical Analysis } \\
\cline { 2 - 6 } & $\begin{array}{c}\text { Hand } \\
\text { Game } \\
\text { Addition }\end{array}$ & $\begin{array}{c}\text { Hand } \\
\text { Game } \\
\text { Subtraction }\end{array}$ & $\begin{array}{c}\text { Lift the Bowl } \\
\text { Addition }\end{array}$ & $\begin{array}{c}\text { Lift the Bowl } \\
\text { Subtraction }\end{array}$ & $\begin{array}{c}\text { Kabuuang } \\
\text { Kasanayan sa } \\
\text { Numeracy }\end{array}$ \\
\hline Level of Significance & $5 \%$ & $5 \%$ & $5 \%$ & $5 \%$ & $5 \%$ \\
Degrees of Freedom & 27 & 27 & 27 & 27 & 27 \\
Critical $t$ - value & 2.052 & 2.052 & 2.052 & 2.052 & 2.052 \\
Computed $t$ - value & 6.0531 & 4.2451 & 6.5279 & 4.4016 & 6.9997 \\
Decision on $\mathrm{H}_{\mathrm{o}}$ & Reject & Reject & Reject & Reject & Reject \\
Conclusion & Significant & Significant & Significant & Significant & Significant \\
\hline
\end{tabular}

Mahalaga ang kabuuang kasanayan sa numeracy ayon sa pre-test at posttest ng mga mag- aaral. Makikita sa talahanayan na ang computed t- value of 6.9997 ay mas mataas kaysa sa critical $t$-value of 2.052 ayon sa $5 \%$ na antas ng pagpapahalaga sa degrees of freedom na 27 na ang ibig sabihin ay rejek ang null hipotesis.

Sa Hand Game Addition, ang pagkakaiba sa antas sa numeracy ng mga mag- aaral ay may mahalagang pagkakaiba. Makikita sa talahanayan na ang computed $t$-value ay 6.0531 ay mas mataas sa critical $t$-value of 2.052 ayon 5\% antas ng pagpapahalaga sa degree of freedom na 27 na ang ibig sabhin ay rejek ay null hipotesis. 
Para sa Hand Game Subtraction, ang pagkakaiba sa antas sa numeracy ng mga mag-aaral ay may mahalagang pagkakaiba. Makikita na ang computed $t$-value na 4.2451 ay mas mataas sa critical $t$-value of 2.052 ayon sa 5\% antas ng pagpapahalaga sa degree of freedom na 27 na ang ibig sabihin ay rejek ang null hipotesis.

Sa Lift the Bowl Addition, ang pagkakaiba sa antas sa numeracy ng mga mag- aaral ay may mahalagang pagkakaiba. Mapapansin na ang computed $t$ - value of 6.5279 ay mas mataaas kaysa sa critical $t$-value of 2.052 ayon sa 5\% na antas ng pagpapahalaga sa degree of freedom na 27 na ang ibig sabihin ay rejek ang null hipotesis.

Para naman sa Lift the Bowl Subtraction, ang pagkakaiba sa antas sa numeracy ng mga mag- aaral ay may mahalagang pagkakaiba. Ang computed $t$ - value ay 4.4016 mas mataas kaysa sa critical $t$-value na 2.052 ayon sa $5 \%$ na antas ng pagpapahalaga sa degree of freedon na 27 na ang ibig sabihin ay rejek ang null hipotesis batay sa pre-test at posttest.

Para naman sa Lift the Bowl Subtraction, ang pagkakaiba sa antas sa Numeracy ng mga mag- aaral ay may mahalagang pagkakaiba. Ang computed $t$ - value ay 4.4016 mas matas kaysa sa critical $t$-value na 2.052 ayon sa $5 \%$ na antas ng pagpapahalaga sa degree of freedon na 27 na ang ibig sabihin ay rejek ang null hipotesis batay sa pre-test at posttest.

Ito’y nagpapakita lamang na epektibo ang ginamit na estratehiya ng guro sa pagtuturo ng Numeracy. Masasabing mabisa ang paggamit ng laro bilang estratehiya dahil tumaas ang antas ng kasanayan sa post test. Dahil sa paggamit ng estratehiyang laro na mas natutong mag-add at magsubtract ang mga bata samantalang sa pre-test walang ginamit na estratehiya, kaya naman ang kanilang antas ng kasanayan ay mababa.

Mas Mabisa ang paggamit ng mga makabagong estratehiya kaysa sa paggamit ng mga tradisyunal na pamamaraan ng pagtuturo. Mas mataas ang nakuhang marka ng mga batang ginamitan ng mga makabagong estratehiya kaysa sa mga batang ginagamitan ng "time tests" at "flashcards" (Boso, 2011).

\subsection{Mungkahing gawain sa pagpapaunlad ng kasanayan ng mga kinder sa numeracy}

Isang sanayang aklat ang ginawa ng mananaliksik upang makatulong na mapaunlad at mapataas ang kasanayan ng mga mag-aaral sa Numeracy. Ang ginawang sanayang aklat ay maaaring gamitin ng mga guro sa kanilang pagtuturo ng Addition at Subtraction. Ito rin ay maaaring gamitin ng mag-aaral sa kindergarten upang maging kawili-wili ang kanilang mga aralin tungkol sa addition at subtraction. Makatutulong ang ginawang sanayang aklat sa mga guro sa pamamagitan ng paggamit nito sa kanyang pagtuturo. Higit itong makatutulong sa mga mag-aaral sa pamamagitan ng pagsagot sa sanayang aklat sa pamamaraang laro na isang kawili-wiling gawain sa kanila.

\section{Kongklusyon at rekomendasyon}

Batay sa mga natuklasan nabuo ang sumusunod: Ang antas ng kasanayan ng mga mag-aaral sa Hand game Addition, Hand Game Subtraction, Lift the Bowl Addition at Lift the Bowl Subtraction ay lahat nasa lebel na Beginning. Naging epektibo ang resulta ng pagtuturo ng numeracy gamit ang Hand Game Addition, Hand Game Subtraction, Lift the Bowl Addition at Lift the Bowl Subtraction sa mga mag-aral sa Kindergarten. May mahalagang pagkakaiba ang antas ng kasanayan sa numeracy ng mga mag-aaral ayon sa pre-test at posttest. Mas tumaas ang antas ng kasanayan sa numeracy ng mga mag-aaral gamit ang mga estratehiyang Hand Game at Lift the Bowl sa pagututuro ng Numeracy sa mga mag-aaral sa Kindergarten. Sanayang aklat ang binuo para sa pagtuturo ng numeracy sa Kindergarten.

Inirerekomenda ang mga sumusunod: Sikapin ng mga guro sa Kindergarten na mapataas ang kasanayan ng mga mag-aaral sa Numeracy sa pamamagitan ng pagpukaw ng kanilang interes sa tulong ng paggamit ng iba-t ibang dulog at estratehiya sa pagtuturo. Mahikayat ang mga guro na gumawa ng mga kagamitang pampagtuturo tulad ng modyul, kalipunan ng mga babasahin at iba pang mga laro na makatutulong sa paglinang ng kasanayan 
ng mga mag-aaral hanggang sa pinakamataas na antas ng komprehensyon sa Numeracy. Sa pagpapataas ng lebel ng kasanayan sa Numeracy ng mga mag-aaral sa Kindergarten, linangin ang mataas na antas ng kognitibong kaisipan ng bawat mag-aaral sa tulong ng mga gawaing tumutugon sa pangangailangan intelektwal ng mga mag-aaral. Magamit ang panukala sa pag-aaral na ito sa mga guro sa Kindergarten upang mas malinang ang kasanayan ng mga bata sa Numeracy. Maaaring magsagawa ng pag-aaral tungkol sa kahandaan ng mga bata sa Matematika upang matukoy ang ilang pang salik na nakakaapekto sa antas ng kasanayan sa Numeracy.

\section{Talasanggunian}

Belvez, P. M. (2005). Ang sining at agham ng pagtuturo. Quezon City: Rex Publishing. Boso, A. (2011). Math fact strategies research project. Institute of Education Sciences.

Code of Ethics for Professional Teachers Article 4: A Teacher and the Profession.

DepEd Order No. 37 S. (2011). Policies and guidelines on the implementation of the universal kindergarten education for SY 2011-2012.

$\mathrm{Ku}, \mathrm{O}$., Chen S., \& Wu, D. (2014). The effects of game-based learning on mathematical confidence and performance: High ability vs. low ability. Educational Technology \& Society, 17, 65-78.

Nolasco, R. D. (2012). MTB-MLE: Far beyond Aquino administration. Philippine Daily Inquirer. Marso 17, 2012

Orfiano, P. A. (2013). Early Childhood Care and Development (ECCD). The Modern Teacher, 62.

Sandigan, N. G. (2010). The effect of multimedia in teaching Mathematics IV (Unpublished masteral thesis). University of Saint Anthony. Iriga City.

Tindugan, S. M. (2014). Kaungkapan at kabisaan ng mga mungkahing istratehiya sa pagtuturo ng mga tekstong letirari sa antas tersarya. Lungsod ng Legazpi.

Vargas, T. H. (1989). Critical thinking skills of students in Mathematics III: Factor correlates of teaching strategies (Unpublished masteral thesis). Sorsogon State College. Sorsogon City. 
Huevos, M., \& Marbella, F. 